\title{
El conflicto de Guyana - Alcan y la nacionalización de Demba
}

\begin{abstract}
NORMAN GIRVAN es profesor de Economía en la Universidad de West Indies en Jamaica y miembro académico del Instituto de Relaciones Internacionales de la Universidad de Chile, donde fue investigador visitante desde octubre de 1969 a enero de 1970. Es autor de Foreign Capital and Economic Underdevelopment in Jamaica (1972); The Caribbean Bauxite Industry (1967) y Copper in Chile: a study in conflict between corporate and national economy (en preparación, 1972). Todos estos libros han sido publicados por el Institute of Social and Economic Research, University of West Indies, Jamaica. En los años 1970 y 1971 fue asesor de bauxita del gobierno de Guyana y miembro del equipo de gobierno que negoció con Alcan Aluminium Ltd., para conseguir una mayor participación en su subsidiaria de bauxita en Guyana.
\end{abstract}

No se puede ser un pais independiente y ser un satélite económico, no tan sólo de otra nación, sino de una corporación privada.

FORBES BURNHAM, Primer Ministro de Guyana.

El 23 de febrero último, el Primer Ministro de Guyana Forbes Burnham anunció -en la que ha sido calificada como una declaración trascendental- la decisión de su gobierno de nacionalizar la Demerara Bauxite Company (DEMBA). DEMBA es, por gran margen, la más grande de las dos empresas de bauxita del país y la principal empresa generadora de divisas e ingresos fiscales en Guyana; constituye también la mayor explotación de bauxita perteneciente a Alcan Aluminium Limited, una gigantesca compañia multinacional de aluminio con un capital de $\$ 2.100$ millones de dólares canadienses y una venta anual de $\$ 1.300$ millones en 1969.

Por lo tanto, el significado de la decisión del Gobierno va mucho más lejos que el mero fracaso de las negociaciones entre éste y la compañía, que condujo al gobierno a aplicar la sanción extrema de una 
nación soberana. En último término, esta decisión implicó un cambio fundamental en la organización de la economía guyana y constituyó la primera desviación concreta, por parte de un gobierno caribeño del Commonwealth, de la política de acomodación al capital externo, que ha sido la tendencia de la política económica de postguerra en la región. Políticamente, constituye la consecuencia más significativa hasta la fecha de la presión hacia la descolonización económica ejercida por la población de la región frente a los gobiernos del Caribe. Como un resultado directo, es probable que la confrontación entre el pueblo y el gobierno, que hasta ahora ha sido la manifestación más visible de esta presión, se transfiera en parte a la relación entre el gobierno de Guyana y los gobiernos del Atlántico Norte y, posiblemente, a sus relaciones con otros gobiernos del Caribe. Pocas dudas existen, por lo tanto, de que la declaración del 23 de febrero marcó un cambio de rumbo en la política económica del Caribe.

Lo que es más, existe la impresión de que la decisión del gobierno no fue el resultado accidental del punto muerto a que llegaron las negociaciones, sino una acción deliberada emprendida con pleno conocimiento tanto de las implicaciones económicas como de las consecuencias políticas de la medida. Retrospectivamente, esta acción puede concebirse como parte del desarrollo lógico de la política externa y doméstica del gobierno desde que, hace más de un año, decidió establecer una "república cooperativa". Desde entonces el sistema político de Guyana se ha caracterizado por una continua tensión entre la retórica y la acción. La política actual, pues, se puede explicar en gran medida por la necesidad que tiene el gobierno de disminuir esa tensión. Sin embargo, al resolverla, se ha creado una nueva tensión entre la necesidad de implementar las nuevas polfticas por una parte $y$, por la otra, un sistema político obsoleto basado en "gobierno" y "oposición", sistema que en Guyana se ve entrabado por la fragmentación de sus dos componentes por causa de diferencias raciales. La medida en que la política guyanesa responda constructivamente a estas tensiones $-\mathrm{y}$ a la necesidad de movilizar a la población en defensa de sus intereses económicos contra el capital externo- determinará no sólo el éxito relativo de la nacionalización de DEMBA, sino también la dirección del desarrollo futuro de Guyana y, a través de ello, el curso de los acontecimientos en áreas más amplias del Garibe. Esto ya debe ser claro para los intereses imperiales del Atlántico Norte, si es que no lo es todavía para los pueblos caribeños.

Habiendo señalado los problemas más amplios planteados por la nacionalización, es necesario poner en claro que este trabajo no in- 
tenta analizar ninguno de ellos en detalle. Se trata más bien de ensayar un análisis preliminar de la política económica que determinó las relaciones entre ALCAN y Guyana desde el inicio de las mismas, hace más de cincuenta años. En otras palabras, este trabajo procura exponer la lógica interna de los hechos que condujeron a la nacionalización de DEMBA, en el contexto de la relación estructural entre las necesidades económicas de una compañía multinacional y la política económica de un país del Caribe. Por otra parte, el autor de este trabajo estuvo personalmente involucrado en los sucesos que llevaron a la nacionalización, por lo que es necesario empezar por declarar que el análisis está influido necesariamente por el privilegio, la responsabilidad y las deficiencias en objetividad y desapego, que son el resultado necesario de haber participado personalmente. ${ }^{1}$

El trabajo presenta en primer lugar, una breve historia de la responsabilidad de ALCAN por el subdesarrollo económico de Guyana a través de DEMBA, para mostrar el incipiente conflicto de intereses que fue siempre una característica de la relación ALCAN-Guyana. Después se discute el preludio a las negociaciones para hacer notar sus conexiones, tanto con el histórico choque de intereses subsiguiente, como con sucesos políticos más recientes en Guyana y en el Caribe. Sigue un análisis de las negociaciones entre DEMBA-ALCAN y el gobierno, que comenzaron en diciembre de 1970 y fueron interrumpidas en febrero. Finalmente, hay algunos comentarios acerca de la decisión de nacionalizar y sus consecuencias inmediatas.

\section{LA HISTORIA}

La Demerara Bauxite Company. se estableció en Guyana Británica en 1916 para mantener y trabajar un número significativo de concesiones de bauxita que habían sido adquiridas por la Aluminium Company of America en la colonia británica a lo largo de los tres años precedentes. La bauxita había sido descubierta en las riberas del rio Demerara por el Geological Survey Department de la colonia en 1910. No tuvieron éxito los esfuerzos para interesar a compañías británicas y europeas en la explotación de los depósitos, y en 1913 el descubrimiento llamó la atención de Arthur Vining Davis, presidente de ALCOA. Davis envió a Mackenzie a la Guyana Británica para apoderarse de la mayor cantidad de bauxita en el menor tiem-

'Las circunstancias de la temprana historia de ALCOA en Guyana Británica han sido expuestas en detalle en mi trabajo: "The Denationalization of Caribbean Bauxite: Alcoa in Guyana." New Forld Quarterly, Jamaica, Vol. 5, Nr. 3, 1971. 
po posible, bajo el pretexto de comprar tierras para una plantación de citrus. Hacia 1916, ALCOA, a través de sus agentes, había aseguraclo para sí el grueso de los ricos depósitos de Guyana Británica susceptibles de transporte fluvial a través del río Demerara.

El interés de ALCOA consistía en asegurarse una fuente fija y confiable de bauxita para alimentar su producción de aluminio en los Estados Unidos. La compañía había sido formada en 1888 para trabajar la patente Hall para la reducción electrolítica del aluminio, y desde entonces había crecido, integrándose verticalmente con la 'construcción de una planta de alúmina en East St. Louis, y la adquisición de minas de bauxita en Georgia y Arkansas. Hacia la época de la primera guerra mundial, la compañía buscaba fuentes extranjeras de bauxita, principalmente en las Guyanas Holandesa y Británica, con el propósito de aumentar sus reservas domésticas y de reservarse la bauxita extranjera frente a las compañías de aluminio competidoras. De este modo, desde la partida, la bauxita de Gurana y Surinam formó parte de un sistema corporativo que confinaba esos países a la producción de la materia prima y reservaba a Norteamérica el procesamiento, la manufactura y la fabricación del producto. Esto también significaba que el excedente producido por la inclustria pertenecía a ALCOA y era utilizado por ella, y que la inclustria de la bauxita estaba sujeta al completo control extranjero.

Desde un comienzo se hizo manifiesto también un conflicto entre las autoridades cle Guyana Británica y la compañía. La posibilidad de vincular los recursos hidroeléctricos de la colonia a sus yacimientos de bauxita para hacer posible el desarrollo de una industria de aluminio rentable era la principal preocupación de los funcionarios clel servicio civil, especialmente Sir John Harrison, el director de Geological Survey. En esa época, sin embargo, eran pocos los funcionarios que hacían alguna distinción entre los intereses de la colonia y los del Imperio Británico, por lo que su preocupación se expresaba en términos de procurar que las inversiones locales sirvieran a los intereses imperiales. Casi con seguridad fue por esta razón que ALCOA estableció la Demerara Bauxite Company en Guyana Británica, en 1916, e hizo grandes esfuerzos para demostrar que sus directores, empleados y la compañía matriz eran británicos. También, por deferencia a la sensibilidad imperial, prometió construir una planta de alúmina para procesar la bauxita de Guyana Británica ren "suelo británico".

No obstante su personalidad legal y sus promesas, DEMBA llegó a ser una parte vital en el sistema de ALCOA, mientras permanecía clivorciacla de los intereses y de la economía guyanesa. Su bauxita .era enviacla a la planta de ALCOA en East St. Louis; la alúmina 
resultante, a las fundiciones de la compañía cuya energia provenia de hidroplantas pertenecientes a ALCOA, y el aluminio producido de esta manera era utilizado por sus propias plantas manufactureras. La oportunidad de cumplir las promesas efectuadas en forma consistente con los intereses de ALCOA se presentó cuando la compañía incorporó la firma canadiense Aluminium Limited, en 1928, transfiriendo a esta compañía sus vastas plantaciones hidroeléctricas y concesiones en Canadá, como igualmente la mayoría de sus propiedades extranjeras, incluida DEMBBA. Al formar Aluminium Limited $^{2}$, compañía matriz de ALCAN, como una compañía legalmente separada e independiente, los dueños de ALCOA pudieron participar en cartels extranjeros, algo que la ley norteamericana le impedía hacer. $Y$ la promesa hecha a Guyana Británica fue cumplida al construir una planta de alúmina en tierra británica: en Arvida, Canadá, cerca de una fundición abastecida por la energía proveniente de una hidroestación de ALCAN. 3

Desde 1928 hasta 1950 la bauxita de Guyana fue la materia prima sobre la cual se basaba el poder creciente y la posición de ALCAN como compañía de aluminio. Este papel fue especialmente fuerte durante el periodo de la Segunda Guerra Mundial, cuando los gobiernos aliados prestaron U.S.\$173 millones a ALCAN para la expansión de sus instalaciones, a fin de suministrar aluminio a la industria bélica ${ }^{4}$. El grueso de la bauxita para este aumento vino de DEMBA. La bauxita de Guyana, por lo tanto, contribuyó marcadamente a la victoria del poderío angloamericano sobre el germanojaponés. Más específicamente, fue la base sobre la cual las inversiones de ALCAN crecieron de U.S.\$45 millones en 1928 a U.S.\$ 423 en 1950, mientras que su producción de aluminio crecía de virtualmente cero a más de 400.000 toneladas. Para Guyana, esto significó que sus recursos naturales fueron empleados en la inclustrialización de la economía norteamericana y en la acumulación de poder y riqueza de las familias Davis y Mellon. En el intertanto, Guyana permaneció subdesarrollada y los guyaneses pobres y económicamente impotentes. 5

'E1 nombre fue cambiado a Alcan Aluminium Limited en 1960. Esta es la compañia que sostiene la red mundial de las subsidiarias de ALCAN y afiliados.

"Ver H. D. Huggins, Aluminium in Changing Communities, Londres, Andre Deutsch en asociación con el Institute of Social and Economic Research, 1965, p. 35.

'Charlotte Muller, Light Metals Monopoly', AwS Press, Nueva York, 1968, pp. 88-95. Los capítulos II a IV proporcionan una excelente visión de la temprana historia de ALCAN como monopolio.

"Para una discusión más sistemática ver mi trabajo: The Caribbean Bauxite Industry, Institute of Social and Economic Research, Universidad de West Indies, 1967. 
En los años 1950, se desenvolvió lo que resultó ser una segunda fase en la estrategia de ALCAN para Guyana. Esta etapa puede ser expresada en una sola frase: bauxita calcinada. Hay que explicar que la bauxita calcinada se usa principalmente en la industria del acero y en otras inclustrias metálicas para la producción de ladrillos refractarios usados en los altos hornos. Se produce por el sobrecalentamiento de la bauxita, que tiene un contenido de hierro muy bajo, y su valor es superior al de la bauxita de grado metálico pero mucho más bajo que el de la alúmina, que es su mayor competidor como material refractario. Entre los años 1950 y 1960, ALCAN desarrolló una importante planta de bauxita calcinada en DEMBA. Otra planta relativamente pequeña de alúmina fue construida también a fines de la década del 50, para usar algo de los desperdicios de bauxita acumulados en los últimos cincuenta años. Las exportaciones de bauxita seca se mantuvieron con el fin de alimentar la planta de alúmina de Arvida. Sin embargo, progresivamente, ALCAN confió menos y menos en Guyana como fuente de material para su creciente producción de aluminio.

Lo que había sucedido era que ALCAN estaba volviéndose hacia nuevos países productores de bauxita. Entre los años 50 y 60 , Jamaica fue el elegido y fueron construidas allí dos plantas de alúmina con una capacidad combinada de más de un millón de toneladas por año. En la década del 70, Guinea y Australia recibirán los beneficios del favor de la compañía. Mientras los ricos depósitos superficiales de Guyana fueron agotándose progresivamente, como resultado de decenios de explotación, los costos subieron y surgieron grandes depósitos superficiales susceptibles de explotación en otros paises.

Hàbía también un sutil elemento de estrategia política envuelto en los planes de ALCAN para Guyana. Alrededor de 1950, Guyana comenzó a ser lo que se conoce en el lenguaje de las compañías internacionales como un poor investment risk. En 1953 el partido nacionalista-marxista People's Progressive Party, obtuvo una mayoría aplastante en el gobierno bajo una nueva constitución. A los seis meses, los británicos expulsaron al partido del gobierno y anularon la constitución. Subsiguientemente, el movimiento se dividió en dos facciones: una hindú, el Progressive Party, presidido por el doctor Cheddi Jagan, y otra africana, el People's National Congress, encabezado por Mr. Forbes Burnham. Como consecuencia de esto, graves violencias raciales se desarrollaron en los años 60. Era, pues, de gran interés para ALCAN el depender lo menos posible de Guyana. Al mismo tiempo, la compañía tenía inversiones y concesiones de bauxita en el lugar, por lo que pretendían sacar el máximo provecho 
de sus operaciones en el país. Al desarrollar la bauxita calcinada en Guyana, ALCAN se concentraba en un producto que dejaba buenas utilidades pero del cual no dependían las plantas procesadoras y productoras de la compañía en el resto del mundo, como dependen de la bauxita y la alúmina. Así, pues, el desarrollo de la industria de la bauxita en Guyana estaba planeado para minimizar el riesgo de las inversiones de ALCAN y, al mismo tiempo, promover sus utilidades. Como un resultado directo de esto, Guyana continuó siendo excluida de la valiosa producción de aluminio y relegada a una relativamente pequeña producción de alúmina, de modo que la contribución potencial de sus recursos de bauxita al desarrollo del país permaneció considerablemente subutilizada después de más de medio siglo de producción de bauxita.

La producción de DEMBA para el período 1917-1969 fue avaluada por la misma compañía en $G \$ 905$ millones $^{6}$, pero estimaciones alternativas usando precios de bauxita y alúmina más justos para el país hubieran puesto esta cifra en un nivel más alto. Sin embargo incluso si se considera el avalúo de la compañía, los ingresos que realmente recibió Guyana ascendieron a un 39 por ciento de esa suma como máximo ${ }^{7}$. Esto es así porque todos los dividendos fueron pagados a accionistas extranjeros y la mayoría de los insumos y equipos eran importados $y$, por lo tanto, pagados a proveedores extranjeros. Lo que es más, una completa evaluación de la contribución de DEMBA, o más bien de su falta de contribución a la economía de Guyana, debe tomar en cuenta el valor agregado en el extranjero al transformar la bauxita y la alúmina guyanesa en aluminio. Mientras el valor medio de la bauxita de Guyana en 1960 era de G\$18 por tonelada (de 2.240 libras), la alúmina producida de ésta podía valer $\$ 65$ y el aluminio derivado más de $\$ 250$. Si se considera que los embarques de bauxita de DEMBA para 1917-1969 sumaban cerca de 50 millones de toneladas y el de alúmina 22 millones de toneladas, se podrá concebir el volumen que alcanzó la transferencia de ingresos de Guyana a Norteamérica durante el período.

\section{EL PRELUdio}

El analista encuentra dificultades para localizar algún hecho en la serie de sucesos que llevaron al gobierno a buscar participación en DEMBA en 1970. Probablemente, el punto más conveniente para co-

\footnotetext{
${ }^{6}$ Radioemitido por J. G. Campbell, presidente de DEMBBA, el 26 de agosto de 1970.

'De $A$ Guyanese version of Demba's Record, por Economist, Guyana Graphic, 27 de agosto de 1970 , p. 1 .
} 
menzar es la resolución adaptada por el People's National Congress, el partido gobernante, en su convención anual llevada a cabo en abril de 1970. Esta resolución pedía al gobierno, por sí mismo o en asociación con cooperativas, que poseyera un 51 por ciento de las acciones en inversiones para la explotación de los recursos forestales y mineros del país.

Sin embargo, esta resolución, que fue usada después con frecuencia por el gobierno como un mandato, sólo puede ser adecuadamente entendida tomando en cuenta los antecedentes de la historia de DEMBA en Guyana y la política interna del país. Como hemos visto, los términos en los cuales DEMBA fue establecida en Guyana la convierten en una operación de expropiación extranjera $y$ el comportamiento ulterior de la compañía confirma este punto de vista. No solamente la bauxita de Guyana fue usada para industrializar y desarrollar Canaclá y los Estados Unidos antes que Guyana, sino también la comunidad minera de DEMBA, situada a unas sesenta millas sobre el río Demerara, se transformó en una llaga colonial abierta en el cuerpo de la sociedad guyanesa. En efecto, la comunidad Mackenzie-Widmar combinaba las características de supremacía blanca y segregación residencial y recreacional con las más deplorables condiciones de vivienda para los trabajadores y sus. dependientes, coexistiendo cara a cara con el agradable nivel de vida de los blancos. Mackenzie llegó a ser un hombre legendario en Guyana, significando salarios relativamente altos al mismo tiempo. que elevado nivel de alcoholismo, relaciones inestables entre hombres y mujeres y una alta incidencia de enfermedades venéreas, una gran rotación de trabajadores y un estado constante de inquietud laboral, caracterizado por continuas huelgas ${ }^{8}$. A pesar de que DEMBA empleaba una porción relativamente pequeña de la fuerza de trabajo de Guyana, existían pocos guyaneses que nunca hubieran trabajado o vivido allí, o que no tuvieran parientes que hubieran trabajado o vivido en el lugar, y que no compartieran esa relación de amor-odio con DEMBA, debida a la combinación de altos salarios con condiciones repugnantes de vida y trabajo.

En seguida, debe recalcarse que esta comunidad existía en el contexto de una Guyana que desde principios de los años 50 ha sido marcada por una fiebre de activismo político, caracterizado por ideologías nacionalistas y socialistas y por una fuerte conciencia racial. Realmente, reconociendo la primacía del elemento racial en

${ }^{8}$ Esto está bien analizado por Maurice St. Pierre, Industrial Unrest in Guyanese Mining Community, Critchlow Labour College, Georgetown, 1969, y Cedric Grant,. Company Towns in the Caribbean, en Caribbean Studies, abril de $197 \mathrm{I}$. Puerto Rico. 
la política de Guyana desde fines de la década clel 50, es fácil olvidar el hecho de que todos los sectores de la población guyanesa han sido radicalizados por el People's Progressive Party y por la intervención británica en 1953, y que ambas secciones del movimiento nacional retuvieron una ideología nacionalista y socialista aún después de dividirse. Por lo demás, a pesar de que la encarnizada lucha de 1960 apartó a la población de un camino de confrontación con el poder imperial, implicó un alto grado de movilización de africanos e hindúes en defensa de evidentes intereses de grupo, una movilización de gran potencial revolucionario para el futuro.

El tercer factor que debe tomarse en cuenta es la desafección entre grandes sectores de la población del Caribe desde que la implantación de la independencia política resultó ser incapaz de traer consigo el fin de la dominación económica blanca y de la miseria económica de los negros. Este desapego es más notorio en Jamaica, doncle se manifiesta en sangrientas confrontaciones diarias entre la policía y el pueblo, y en Trinidad, donde las demostraciones del black power casi botaron al gobierno en 1970. Ninguno de estos problemas deja de estar presente en Guyana y allí, así como en el resto de la región, presentan al régimen un angustioso dilema: cómo puede un régimen político que ha llegado al poder como resultado de un proceso de movilización popular, por un lado, y de acomodación a intereses políticos y económicos de origen externo por otro, enfrentar una situación en la cual los dos extremos se vuelven cada vez más irreconciliables. En Guyana los medios elegidos fueron el establecimiento de una república cooperativa. en febrero de 1970 con el objetivo de "hacer del hombre pequeño un hombre verdadero", y el instrumento elegido fue la cooperativa. Considerando que desde el principio el sector cooperativo no fue utilizado para desafiar el poder extranjero blanco, era obvio que el proceso de descolonización económica no podía ir muy lejos sin una confrontación de ese tipo. En los meses de abril y mayo, el Primer MLinistro comenzó a hablar de las intenciones del gobierno de buscar una participación significativa en la industria de bauxita y para fines de mayo pareció que el gobierno, de hecho, estaba orientándose de acuerdo con los intereses de la mayoría.

En este punto, dos cosas se hicieron evidentes. Primero, el gobierno había estado hasta entonces actuando según la lógica de su posición oficial acerca del control nacional sobre los recursos naturales, al tratar de adueñarse de la industria con mayores recursos del país. Esta prontitud para responder a la lógica cle su posición iba a presagiar la subsiguiente decisión de nacionalizar en vista de la intransigencia de ALCAN. Lo segundo fue la intención gubernamen- 
tal de equiparse lo mejor posible con conocimiento técnico y preparación antes de comenzar las negociaciones con las compañías de bauxita. Se formó un equipo que recolectó expertos al servicio del gobierno en los campos de la geología, economía, impuestos, estadísticas $y$ contabilidad. Fueron también utilizados como asesores dos académicos de West India que habían trabajado en los aspectos económicos y políticos de la bauxita. El gobierno dio a este equipo libertad para discutir, evaluair y recomendar diversas estrategias alternativas para la participación estatal en la empresa. Y a pesar de que las compañías estaban listas para comenzar las negociaciones desde abril, la seriedad del gobierno se manifestó por su intención de esperar meses mientras sus técnicos se preparaban y adquirían el conocimiento y experiencia necesarios para las negociaciones.

Al pasar los meses, la aprensión inicial de estos hombres y la enormidad de la tarea encomendada a ellos por los políticos, fue reemplazada por una actitud de confianza creciente y de excitación ante la perspectiva de aceptar responsabilidad para una industria de bauxita nacionalmente controlada. Gracias a la evaluación de los convenios de participación de Chile y Zambia, estos funcionarios y el gobierno vieron con claridad muchas cosas. Entre ellas, que las compañías mineras multinacionales en estos países sólo concedieron a los gobiernos intereses mayoritarios a cambio de ciertas concesiones significativas para el país. Primeramente, las compañías retenían el control sobre las operaciones por medio de contratos de administración y una participación minoritaria en el capital accionario de la empresa, lo que les daba la posibilidad de llenar los puestos directivos claves y un poder de veto sobre las decisiones importantes relacionadas con las políticas de producción y financieras. En segundo lugar, las compañías lograban una posición financiera superior como resultado de la participación gubernamental en ellas, al obtener que el gobierno disminuyera los impuestos y controles sobre las utilidades remitidas al exterior, al asegurar la expansión financiada por el gobierno o por terceras partes, $y$ al asegurarse un favorable avalúo de los bienes por adquirir y los medios para pagar esas inversiones. Por ejemplo, al asegurarse el avalúo de sus inversiones al valor de mercado, con pagos durante un período de tiempo relativamente corto y sujetos a tasas comerciales de interés, la participación gubernamental podía ser financieramente favorable para las compañías, $y$, al mismo tiempo, desfavorable para el gobierno. ${ }^{9}$

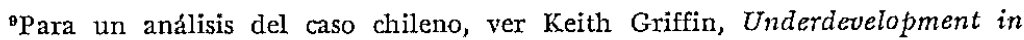
Spanish America, Londres, G. Allen y Unwin, 1969, pp. 149-173, y de este autor Gopper in Chile: a Study in Conflict between Corporate and National Economy, ISER, Mona, en prensa. 
Por lo tanto, el gobierno de Guyana llegó a la convicción de que un acuerdo que contemplara los aspectos financieros y de control de los modelos de Chile y Zambia sería tanto técnicamente como políticamente peligroso. Esta convicción iba a dar pábulo al más importante de los puntos planteados más adelante como de carácter no negociable por parte del gobierno en las conversaciones con DEMBA. 10

Pero habiendo decidido esto, el próximo paso lógico tenía que ser encarado y era éste: suponer que la compañía, enfrentada a un gobierno que no quería negociar en los puntos más importantes, decidiera no llegar a acuerdos; suponer que la compañía retrocediera, borrara Guyana, o simplemente rehusara aceptar la posición del gobierno. Entonces Guyana se vería enfrentada a la tarea de manejar la industria por sí misma, sea porque la compañía hubiera retrocedido, o porque el gobierno hubiera tenido que imponer una solución.

En otras palabras, el gobierno tendría que estar preparado para nacionalizar como parte de sus preparativos para participar. De otro modo, el gobierno hubiera estado negociando desde una posición de debilidad y las negociaciones difícilmente hubiesen sido tomadas en serio.

En efecto, lo que sucedió en los meses precedentes a las negociaciones fue la emergencia y crecimiento de una preparación psicológica para la nacionalización, tanto por parte de los políticos como de los técnicos. $Y$ en forma inevitable, esto condujo a la identificación y preparación de los pasos físicos necesarios para la implementación de cualquier decisión de nacionalizar. Desde los comienzos, se llevó a cabo una evaluación preliminar sobre la capacidad de Guyana para contratar y atraer personal independientemente. Algo que quedó en evidencia como resultado de este ejercicio fue la convicción de que el mundo tenía la suficiente necesidad de los productos de la industria guyanesa de bauxista, y el gobierno la suficiente experiencia para hacer una industria comercialmente viable e independiente de ALCAN. Lo segundo fue que, a pesar de esto, no había seguridad absoluta de que Guyana pudiera mantener sus exportaciones, especialmente en el corto plazo, a un nivel suficiente para evitar distorsiones serias. Más allá de un cierto punto, por consiguiente, la determinación de nacionalizar habría tenido que ser un paso en el vacio y que podría haberse dado si tanto el gobierno

${ }^{20}$ Ver también de este autor: Bauxite, why we need to nationalize and how to do it. New World Pamphlet, Nr. 6, Jamaica, 1971. Reimpreso en N. Girvan y O. Jefferson (Eds.). Readings in the Political Economy of the Caribbean, Mona, Jamaica, New World Group Ltd., 1971. 
como el pueblo no hubieran tenido la confianza en su capacidad para manejar la situación y para hacer sacrificios que son los que distinguen al hombre del niño y al ser independiente del colonizado.

Fue alrededor de este punto donde se desarrollaron las más difíciles discusiones a nivel técnico. En último término, el dilema fue resuelto por los políticos, invocando la más simple de las razones políticas: "Yo prefiero" - dijo el Primer Ministro- "tomar posesión de ALCAN antes que perder el pueblo de Guyana" y, en otra ocasión, "Camarada, es mejor morir cle pie que vivir de rodillas". En diciembre, época en que comenzaron las negociaciones, había pocos representantes gubernamentales que no reconocieran que el gobiemo podría tener que enfrentar la posibilidad de aplicar la medida más extrema, la expropiación, preconizada por el doctor Jagan y su P.P.P. y por el doctor Thomas del Ratoon Group, a pesar de que en ese momento el gobierno no podía aceptar públicamente esa posibilidad, por encontrarse comprometido con la idea de negociar. Como consecuencia de esto, se iniciaron serios estudios sobre las implicaciones que una expropiación tendría sobre el capital de trabajo, los ingresos en divisa, abastecimientos y mercados de la empresa, como también de sus implicaciones legislativas.

\section{LAS NEGOCIACIONES}

La táctica del gobierno fue, primeramente, notificar a la compañía la fecha propuesta para comenzar las negociaciones, llegando al acuerdo de reunirse el 7 de diciembre. El paso siguiente consistió en establecer las condiciones no negociables, tanto para la compañía como para el público; antes de que las conversaciones se iniciaran. Al informar a la compañía de estos puntos a través de una carta privada, el contenido de la cual fue revelado por ALCAN, el gobierno buscaba fortalecer a su equipo negociador, colocándolo en condiciones de negarse a aceptar proposiciones que estuvieran en conflicto con los aspectos no negociables. Al poner esos puntos en conocimiento del público a través de un radiocomunicado del Primer Ministro el 28 de noviembre de 1970, el gobierno buscaba un consenso nacional que pudiera dar legitimidad política a su posición frente a la compañía y al mundo exterior. Estos puntos no negociables eran los siguientes:

1. La participación del gobierno debería ser mayoritaria.

2. Dicha participación se lograría por medio de la compra de acciones de la compañía.

3. El avalúo del capital para los efectos de adquirir esa mayo- 
ría de acciones sería su valor contabilizado para fines impositivos. 4. El pago de las acciones adquiridas por el gobierno se efectuaría con cargo a la participación estatal en las futuras utilidades de la empresa, después del pago de los impuestos.

5. La participación del gobierno en las acciones de la compañía debería llevar consigo el control efectivo propio de una participación mayoritaria.

6. Cualquiera que fuera la fecha del acuerdo a que pudieran llegar el gobierno y la compañía, la fecha efectiva de adquisición sería el 1 o de enero de 1971.

Un análisis de estas condiciones no negociables muestra que el propósito del gobierno era adquirir la mayoría de las acciones, asegurándose al mismo tiempo ciertas condiciones favorables, que en los acuerdos de Zambia y Chile habían sido ideadas para frustrar. el propósito fundamental de la participación mayoritaria. Al referirse a "un efectivo control", el gobierno estaba en realidad notificando a ALCAN que no aceptaría ningún contrato de dirección ni ningún derecho de la minoría que pudiese paralizar su poder de decisión como accionistas mayoritarios. Al exigir que se considerara el valor contabilizado del capital para fines tributarios, el gobierno estaba rechazando un avalúo con base en el valor de reemplazo o en el: valor de mercado, que podría resultar en que el gobierno tuviera que pagar dos o tres veces el monto del avalúo tributario, una diferencia de cientos de millones de dólares. Al especificar que los pagos se harían con cargo a las utilidades futuras, éste estaba diciendo dos cosas. La primera, que como resultado de esta operación su situación financiera no podría empeorar. Esto era una lección extraída directamente de las experiencias de Chile y Zambia, donde los pagos fueron hechos a través de bonos del estado, emitidos cuando el precio del cobre estaba muy alto y la participación estatal en los beneficios era más que suficiente al comienzo para cubrir los pagos, situación que cambió más tarde al bajar el precio del cobre, lo que enfrentó a estos gobiernos con la perspectiva de pagar más de lo que estaban percibiendo. En segundo lugar, al hacer depender los pagos de las utilidades futuras, el gobierno estaba suministrando a ALCAN un estímulo para cooperar como accionista minoritario, y asegurarse de que las ganancias futuras fueran reales.

Los puntos no negociables, sin embargo, dejaban todavía considerable campo para regateos y concesiones por ambos lados. La participación del gobierno podía, en teoría, establecerse en cualquier punto entre los límites del 99 y el 51 por ciento, como resultado de las negociaciones. La proporción de la participación estatal en 
las utilidades futuras, asignada para el pago de las acciones, deducidos los impuestos, era negociable. A pesar de que el gobierno insistiera en un control efectivo, el área y la extensión de la intervención de ALCAN en la administración y decisiones, eran también negociables. Puesto que el problema de la comercialización de los productos de DEMBA no fue mencionado como no negociable, quedó abierto el camino para que ALCAN negociara contratos de compra a largo plazo para estos productos como parte del acuerdo.

En la semana que medió entre la declaración de los puntos no negociables y el comienzo de las conversaciones con la compañía, hubo reacciones tanto del pueblo de Guyana como de ALCAN. De parte del público, surgió un apoyo claro y mayoritario en favor de los principios de propiedad y control de la bauxita por parte del gobierno en general y de DEMBA en particular, y de las condiciones establecidas por el gobierno respecto de avalúos y pagos. La única divergencia significativa frente a las proposiciones del gobierno wino de la oposición del P.P.P. y de un economista de la Universiıdad, quienes exigieron que el gobierno expropiara, esto es, llegara a la total nacionalización tanto de DEMBA como de la compañía más pequeña Reynolds Guyana Mines. Así, desde el comienzo de las conversaciones, al gobierno le fue posible exponer a la compañía que su posición "era factible de ser criticada, si es que lo era, sólo por su moderación".

La reacción de ALCAN no fue de sorpresa, sino de consternación, frente a lo que ellos percibieron como un repentino y dramático cambio de actitud y de tácticas por parte del gobierno. La compañia anunció que lamentaba "que el gobierno de Guyana hubiera decidido, aparentemente en forma unilateral, cambiar de un modo radical los convenios bajo los cuales la Demerara Bauxite Company había operado en Guyana" 11. La compañía dijo que, sin embargo, se reuniría con el gobierno el 7 de diciembre como estaba previsto, para "buscar la clarificación de los puntos expuestos", asegurando a sus accionistas, empleados y clientes que "salvaguardaría sus intereses" y que si "la provisión de bauxita de grado metálico y de alúmina se veían afectados, ALCAN creía que podían buscar fuentes de recursos alternativas".

Tomada en su conjunto, la reacción de ALCAN reveló algunas características significativas. Para comenzar, existe casi certeza de que la compañía jamás contó con el grado de politización con que el gobierno había teñido el caso al hacer públicas sus proposiciones y al generar un apoyo nacional. Además, ALCAN estaba genuinamente sorprendida de que el gobierno hubiera especificado algunos pun-

${ }^{11}$ Comunicado de prensa -de ALGAN, 30 de noviembre de 1970. 
tos no negociables y se mostró horrorizada de la severidad de sús condiciones, desde su propio punto de vista. En otras palabras, la compañía debía haber esperado que las conversaciones se desarrollaran privadamente, sin publicidad, y sin que ninguna de las dos partes, especialmente el gobierno, hubiera adoptado una posición determinada desde el principio. Por eso, la compañía aclaró al go. bierno y a sus accionistas que su asistencia a las conversaciones del 7 de diciembre no implicaba la aceptación de las condiciones no negociables y además, que estaba preparada para arreglárselas sin los productos de DEMBBA si se llegaba hasta ese extremo. Para todos los efectos, la confrontación entre Guyana y ALCAN empezó antes que las conversaciones se iniciaran.

Esta atmósfera de confrontación era tangible cuando se abrió el debate en la sala de directorio de la Guyana Development Corporation. EI gobierno había reunido un numeroso y variado equipo de negociadores pertenecientes a las capas superiores del servicio gubernamental y del mundo académico. Bajo la dirección del Ministro de Minas y Bosques (entonces ministro sin cartera), incluía al jefe de Estadística, al secretario permanente del Ministerio de Desarrollo Económico, al director de Investigaciones Geológicas, al economista "senior" del Banco de Guyana, a la Guyana Development Corporation y a la Guyana Marketing Corporation, dos asesores especiales y el Presidente de la Unión de Mineros con el Alcalde de Markenburg (la comunidad minera) como observadores. Así, prácticamente no hubo ninguna declaración hecha por el grupo DEMBA-ALCAN, ni ningún aspecto de las operaciones de ALCAN o DEMBA que no fuera de inmediato objeto de estricta investigación y severo escrutinio por parte de algún representante del equipo gubernamental. El grupo de DEMBA resultó estar compuesto exclusivamente por funcionarios de la oficina principal de ALCAN en Montreal, y en un momento determinado llegaron a estar presentes al mismo tiempo el Vicepresidente Financiero de ALCAN, el de la fundición y el de materia prima.

Sería ingenuo pretender que las actitudes expuestas alrededor de la mesa de negociación no reflejaban la amplia confrontación que existe en todas partes entre el Tercer Mundo y el capitalismo. metropolitano. Desde el punto de vista de los representantes de ALCAN, ellos estaban tratando con "nativos" por los cuales sentían profundo desprecio y algunos de ellos no hicieron nada por ocultarlo, mientras otros hacían heroicos esfuerzos que solamente se debilitaron bajo condiciones de agotamiento, fatiga y provocación.

Sin embargo, no hay que poner tanto énfasis en las personalidades envueltas en las negociaciones, puesto que ambos grupos estaban ne- 
gociando bajo instrucciones y ambos representaban fuerzas más importantes y más impersonales. Lo que sucedió en las conversaciones entre el 7 de diciembre y el 20 de febrero ahora pertenece a la historia, y la versión de Guyana, que hasta ahora no ha sido desmentida, fue expuesta por el Primer Ministro en su discurso del 23 de febrero. En efecto, DEMBA-ALCAN nunca estuvo de acuerdo con las condiciones no negociables en su conjunto. La compañía tampoco pretendió regatear seriamente las proposiciones del gobierno. El 15 de diciembre expuso en cambio una contraposición, que fue modificada el 9 de febrero. El elemento básico de esta contraposición era la formación de una sociedad entre el gobierno y ALCAN. El gobierno debería contribuir con $\$ G 50$ millones en efectivo, proporcionados por el Banco Mundial o por otra fuente extraña a ALCAN, y ésta contribuiría con DEMBA. Los $\$ 50$ millones del gobierno serían empleados inmediatamente para financiar la expansión de la producción de bauxita calcinada, y esta expansión sería la única que emprendería la nueva compañía, al menos en los próximos cinco años. La nueva compañía indemnizaría a ALCAN el valor de los inventarios de DIMBA aportados a título de préstamo, principalmente con cargo a su fondo de depreciación.

El significado de esta proposición era que, en vez de participar en DEMBA como hasta ahora, el gobierno deberia reunir $\$ 50$ millones como condición para participar en la nueva empresa. En segundo lugar, estos recursos serían usado para un programa de expansión que había sido planeado y cliseñado por ALCAN y que, significativamente, excluía la posibilidad de expandir la alúmina e iniciar la producción de aluminio. El gobierno tenía que expresar

- su conformidad con este programa antes de entrar en la empresa de bauxita y de haber tenido la oportunidad de apreciar las posibilidades de expansión desde adentro. En tercer lugar, pagar a ALGAN principalmente del fondo de depreciación de la empresa. significaba pagar antes de los impuestos y antes cle las utilidades, y por lo tanto, poner tales pagos en la categoría cle costos deductibles. Así, hubiera utilidades o no, siempre habría que pagar a ALCAN. También, al insistir en pagos provenientes del fondo de depreciación en adición a la distribución obligatoria de las utilidades, la empresa hubiera sido despojada de toda posibilidad de financiar su propia expansión.

Además, ALCAN propuso que la responsabilidad de la gerencia operativa de la nueva compañía residiera en un alto ejecutivo nombrado por ALCAN. Los poderes y autoridad de esta persona estaban definidos tan ampliamente que combinaban en un solo cargo muchos de los poderes normalmente ejercitados por una junta de gerentes y 
por una junta de directores y por lo tanto, este jefe ejecutivo haría, -según las palabras de un colega-, "ver al Zar de Rusia como un demócrata".

Ciertas características de la proposición de ALCAN fueron modificadas en el curso de las conversaciones. Desde la partida, ALCAN propuso que la compañía sería capitalizada en aproximadamente $\$$ G 100 millones, 5l por ciento pertenecientes a ALCAN y el 49 restante al gobierno. Los $\$ 49$ millones a que ascendería la participación del gobierno provendrían de un préstamo del Banco Mundial y se cubrirían por entrega de DEMBA. El resto del valor de DEM$\mathrm{BA}$, aproximadamente $\$ 49$ millones, sería aportado como un préstamo a la nueva compañía, reembolsable en cuotas anuales y con una tasa de interés comercial. Subsiguientemente, en febrero, esto fue modificado como sigue:

1) La compañía sería capitalizada en $\$ 100$ millones, 51 por ciento de Guyana y 49 por ciento de ALCAN.

2) El monto total del capital de DEMBA sería aportado en forma de préstamo.

3) La compañía y sus accionistas no estarían sujetos a impuestos de ningún tipo por parte de Guyana, ni a ninguna restricción relativa a intereses, dividendos $\mathrm{u}$ otros beneficios de sus accionistas.

4) Las utilidades de la compañía después de pagar una pequeña suma por aumentos de capital fijo, y amortización e intereses de sus obligaciones, serían asignadas en un 70 por ciento para el gobierno $y$ en un 30 por ciento para ALCAN.

El efecto de estas modificaciones habría sido que ALCAN recuperara el valor de sus inversiones en DEMBA, con una tasa de interés de valor comercial y que, durante y después de las recuperaciones, se convertiría en accionista en una empresa ampliada con un 49 por ciento de sus acciones. Al no pagar la compañia impuestos de ningún tipo, su participación del 30 por ciento en las utilidades por distribuir, habría sido equivalente a la suma obtenida si hubiera tenido una participación del 50 por ciento después del pago de impuestos. De esta manera, una distribución de los beneficios del 70 y 30 por ciento era totalmente ilusoria y, lo que es más grave, iba unida a una completa abrogación de los derechos soberanos del gobierno en materia tributaria, en lo que respecta a la mayor industria de su economía. Después de todo, ALCAN sacaría mucho más provecho de la empresa a través de amortizaciones y utilidades que lo que había estado obteniendo de DEMBAA, sin invertir un nuevo centavo. Además, habría tenido el control operativo de la empresa y habria impulsado una expansión que no amenazaría las plantas de 
alúmina y aluminio que ALCAN poseía en otras partes, lo que era consistente con la estrategia mundial de ALCAN.

El gobierno, por su lado, habría tenido que aumentar de inmediato la deuda pública en $\$ 50$ millones, habría obtenido un aumento de ingresos relativamente pequeño en la industria, se habría visto obligado a efectuar una expansión que excluía la alúmina y el aluminio, y habria tenido poco o ningún control sobre la empresa.

De este modo, se evidenció que las necesidades de AICAN y las de Guyana expresadas en sus respectivas posiciones, eran irreconciliables. Semanas de largas discusiones no hicieron más que confirmar estas diferencias. De acuerdo a lo dicho por el Primer Ministro:

"Me parece que no tenemos más remedio que declarar que no podemos avanzar más. Hemos ofrecido paz y razones y nos hemos encontrado con irracionalidad. Hemos ofrecido una asociación y se nos ha amenazado con prolongar la dominación. Nuestra elección es o ser hombres o ser ratas".12

En estas circunstancias el gobierno tenía, hablando en general, dos alternativas. Una, podía aceptar las proposiciones de ALCAN en to esencial, en la esperanza de encontrar los \$50 millones para hacer efectiva su participación, y lo que exa más importante, en la esperanza de que tales proposiciones hubieran sido presentadas a la nación guyanesa en consistencia con las condiciones no negociables $y$, por lo tanto, como un triunfo para el gobierno. Afortunadamente, éste tenía un gran respeto por la inteligencia de la población del país.

La única otra opción era, evidentemente, la nacionalización, para la cual el gobiemo estaba psicológicamente preparado y para lo cual se efectuaron preparaciones físicas de un modo u otro durante enero y febrero. Las conversaciones con ALCAN fueron rotas formalmente el 20 de febrero de 1971 y la decisión de nacionalizar anunciada el 23 de febrero de ese año, el primer aniversario de la república cooperativa.

EI problema inmediato que trajo la determinación de nacionalizar no fue operacional sino político. I.a compañía continuó produciendo durante los día siguientes a esta determinación y el equipo extranjero no corrió a 'tomar el próximo aeroplano que los llevara fuera del país. En realidad, esto no fue sorprendente, ya que a ALCAN le convenía extraer la mayor cantidad de bauxita posible hasta el último día, y la compañía no podía encontrar trabajo para 70 expatriados de la industria de la noche a la mañana. ALCAN ha anunciado que desea hacer una entrega ordenada de la empresa

12Del discurso del 29 de febrero de 1971 . 
al gobierno y hasta el momento, no se sabe qué quiere en cambio.

La tarea inmediata fue pasar una ley de nacionalización que, al mismo tiempo, cambiara la constitución de Guyana (hecha en Lancaster House), para sustituir la expresión "razonable compensación" por la de "pronta y adecuada" en la sección correspondiente. a la nacionalización. La posición del gobierno era que el único método de pago razonable debía ser a cuenta de futuras utilidades, puesto que, usando las palabras del Ministro Jack al dirigirse a la Cámara de Comercio de Georgetown: "Guyana es un país pobre, y. somos pobres porque compañías como DEMTBA nos han estado explotando". Para asegurar la aprobación de la ley, el gobierno necesitaba el apoyo de la oposición para obtener la mayoría de dos tercios requerida en la Asamblea Nacional. A pesar de que el partido de oposición, P.P.P., siempre había abogado por la confiscación, éste consideró oportuno exponer importantes quejas, relacionadas. con su exclusión del partido o de sus miembros del sistema político. Después de una semana de intensas discusiones entre el Primer Ministro y el jefe de la oposición, el $1^{9}$ de marzo se llegó a un acuerdo, el así llamado plan de paz de Burnham-Jagan, que sorprendió a muchos en Guyana por el tipo y el número de concesiones hechas. por el gobierno a la oposición. Las características principales fueron:

i) El partido de oposición estaría representado en todas las corporaciones del gobierno, directorios y comités, incluyendo la nueva compañía nacionalizada de bauxita por formarse.

ii) La comisión de elecciones sería reconstituida de acuerdo con la constitución y se le darían mayores poderes.

iii) El doctor Jagan proporcionaría las bases para la creación decomités permanentes contra la discriminación y la corrupción.

iv) El gobierno buscaría una política de empleo justo hacia los. guyaneses entrenados en países del bloque oriental.

v) No se renovaría en junio la parte del Acta Nacional de Seguridad que daba al Ministro del Interior amplios poderes para arrestar y detener a las personas, y el derecho de declarar en estado deemergencia a cualquier parte del país. Tampoco sería negado el derecho a demostraciones pacíficas y se terminaria con la práctica de no otorgar pasaportes a los miembros del P.P.P. que querían viajar al extranjero, incluidos los países socialistas.

vi) El parlamento se reuniría con más frecuencia para discutir mociones y preguntas de la oposición, y el Primer Ministro y el líder de la oposición se reunirían lo menos una vez al mes.

vii) La Asociación de Productores de Arroz, compuesta de representantes elegidos por los productores pero reconocida como simpati- 
zante del P.P.P., sería representada nuevamente en el directorio del mercado del arroz.

viii) Se implantaría una nueva actitud en el cobro de los alquileres de los campesinos de los distritos más pobres. El doctor Jagan había reclamado contra el desmantelamiento de sus viviendas para cobrar los arriendos.

ix) El Gobierno haría arreglos para certificar cuál de los dos sindicatos - uno pro oposición y el otro tradicionalmente ligado al partido de gobierno- era realmente representativo de los trabajaclores del azúcar.

$\mathrm{x})$ Los dirigentes del partido tendrían el derecho de seleccionar sus candidatos a representantes y de revocar sus candidaturas.

Es posible, por supuesto, entender el plan de paz como un alto precio exigido por la oposición a cambio de su apoyo a la ley de nacionalización y pagado reluctantemente por el gobierno. Pero, probablemente, esta interpretación encubre la voluntad efectiva del cloctor Jagan de apoyar la nacionalización y el deseo de Mr. Burnham de llegar a una tregua con la oposición. El doctor Jagan había estado hacía largo tiempo abogando por la nacionalización, y es improbable que hubiese querido pasar a la historia como el hombre que bloqueó la nacionalización de DEMBA, aun habiendo sido menores las concesiones del gobierno. Por el lado de Mr. Burnham, él debe haber estado profundamente consciente de su necesidad de contar con el apoyo de la oposición, no sólo para la aprobación de las medidas legislativas sino también para afrontar el período de dificultades y dislocaciones que tenía por delante, como resultado de la decisión de expropiar.

Para concluir, sólo quisiera hacer tres breves comentarios. Primero, que el éxito de la actuación de Guyana en el futuro no sólo depende de su competencia técnica para organizar y manejar las operaciones de producción, sino también de la medida en que la población como un todo se vea envuelta en el proceso de nation building y en la defensa de sus derechos. Segundo, que lo que sucedió y lo que sucederá en Guyana afectará ciertamente los acontecimientos en Jamaica y Surinam, y los guyaneses están ahora conscientes de su responsabilidad de ser un ejemplo para otros pueblos que en el Caribe demandan la devolución de sus recursos. Y finalmente, tal como los guyaneses, en cierto sentido, han aceptado sus responsabilidad frente a nosotros, nosotros en cambio tenemos la importante responsabilidad de apoyarlos en su empresa en lo que nos sea posible.*

*N. del E. La nacionalización de DEMBA se hizo efectiva, tanto desde el punto de vista jurídico como práctico, el 15 de julio de 1971. 